\title{
Dopant and Self-Diffusion in Extrinsic n-Type Silicon Isotopically Controlled Heterostructures
}

\author{
Hughes H. Silvestri, ${ }^{\mathrm{a}, \mathrm{b}}$ Ian D. Sharp, ${ }^{\mathrm{a}, \mathrm{b}}$ Hartmut A. Bracht, ${ }^{\mathrm{c}}$ Sam P. Nicols, ${ }^{\mathrm{a}, \mathrm{b}}$ Jeff W. Beeman, ${ }^{\mathrm{b}}$ \\ John Hansen, ${ }^{\mathrm{d}}$ Arne Nylandsted-Larsen, ${ }^{\mathrm{d}}$ and Eugene E. Haller ${ }^{\mathrm{a}, \mathrm{b}}$ \\ ${ }^{\mathrm{a}}$ Department of Materials Science and Engineering, University of California, Berkeley, CA94720 \\ bMaterials Science Division, Lawrence Berkeley National Laboratory, Berkeley, CA 94720 \\ ${ }^{\mathrm{c}}$ Institut für Materialphysik, Universität Münster, Germany \\ ${ }^{\mathrm{d}}$ Institute of Physics and Astronomy, University of Aarhus, Denmark
}

\begin{abstract}
We present experimental results of dopant- and self-diffusion in extrinsic silicon doped with As. Multilayers of isotopically controlled ${ }^{28} \mathrm{Si}$ and natural silicon enable simultaneous analysis of ${ }^{30} \mathrm{Si}$ diffusion into the ${ }^{28} \mathrm{Si}$ enriched layers and dopant diffusion throughout the multilayer structure. In order to suppress transient enhanced self- and dopant diffusion caused by ion implantation, we adopted a special approach to dopant introduction. First, an amorphous 250-nm thick Si layer was deposited on top of the $\mathrm{Si}$ isotope heterostructure. Then the dopant ions were implanted to a depth such that all the radiation damage resided inside this amorphous cap layer. These samples were annealed for various times and temperatures to study the impact of As diffusion and doping on Si self-diffusion. The Si self-diffusion coefficient and the dopant diffusivity for various extrinsic n-type conditions were determined over a wide temperature range. We observed increased diffusivities that we attribute to the increase in the concentration of the native defect promoting the diffusion.
\end{abstract}

\section{INTRODUCTION}

The current solution for increased speed in CMOS transistors is the reduction in device size. The main size reduction comes in the form of a shorter channel length. In order to facilitate shorter channel lengths and prevent device breakdown, shallower source and drain regions are needed [1]. Ion implantation of dopants is used to create the very shallow, heavily doped source and drain regions. However, further thermal processing of the device leads to diffusion of the dopant and results in greater junction depth. This problem has highlighted the need for a better understanding of the diffusion of dopants in silicon under extrinsic conditions. An improved understanding of diffusion phenomena in silicon will help to develop physically reasonable models to accurately predict junction depths after thermal processing.

As a step in this direction, we study the diffusion of n-type dopants in Si. Dopant diffusion in silicon is known to be mediated by interstitial silicon atoms and/or lattice vacancies [2]. In the case that these native point defects are charged, their concentration is affected by the position of the Fermi level [3]. In particular, extrinsic n-type (p-type) doping favors negatively (positively) charged defects. In order to understand dopant diffusion in detail, the impact of doping and dopant diffusion on the formation and diffusion of native point defects must be known. This interrelation between self- and dopant diffusion can be studied by utilizing isotopically enriched Si isotope superlattice structures for dopant diffusion experiments.

Recently, the self-diffusion of silicon under electronically intrinsic conditions has been determined over a wide temperature range using isotopically controlled Si heterostructures [4]. 
The temperature dependence of Si self-diffusion coefficients is described by a single activation enthalpy. No evidence of a kink in the temperature dependence has been found which had been suggested on the basis of earlier self-diffusion studies (see references in [4]). The contributions of vacancies and self-interstitials to Si self-diffusion under intrinsic conditions have been obtained from metal diffusion experiments. The sum of these individual contributions to selfdiffusion equals the directly measured Si self-diffusion coefficient. The consistency of the results from metal and self-diffusion experiments provides evidence that self-diffusion is mediated by self-interstitials above $890{ }^{\circ} \mathrm{C}$ and by vacancies below $890{ }^{\circ} \mathrm{C}$. However, recent experiments on $\mathrm{Si}, \mathrm{P}$, and $\mathrm{Sb}$ diffusion under inert and oxidizing ambients seem to indicate that the contribution of self-interstitials and vacancies to self-diffusion may be similar [5].

In order to clarify the inconsistency between the results obtained from metal diffusion and dopant diffusion under oxidation, we investigated the impact of dopant diffusion and doping on $\mathrm{Si}$ self-diffusion. This work demonstrates that dopant diffusion in Si isotope structures not only provides information about the contributions of vacancies and self-interstitials to Si selfdiffusion, but also yields a more detailed insight into the mechanism of dopant diffusion compared to previous studies which were restricted to the analysis of dopant profiles alone [6].

This paper addresses the diffusion of $\mathrm{As}$ in a $\mathrm{Si}$ isotope superlattice structure. Arsenic is known to diffuse via self-interstitials and vacancies in Si. By studying the changes in As and Si diffusion under extrinsic conditions, information about the mechanisms of As diffusion in Si and the charge states of the native defects were obtained. Moreover, the individual contributions of these charged defects to Si self-diffusion were determined.

\section{EXPERIMENTAL}

A multilayer structure consisting of alternating layers of enriched ${ }^{28} \mathrm{Si}$ and natural Si was grown on a Si wafer of natural isotopic composition via ultra-high vacuum chemical vapor deposition at Lawrence Semiconductor Research Laboratories. Five ${ }^{28} \mathrm{Si}$ enriched layers were grown, separated by natural Si layers. Each individual layer was $120 \mathrm{~nm}$ thick.

Ion implantation was chosen as the method to introduce the dopant species into the multilayer structure because of its ability to achieve high doses. However, ion implantation is known to produce interstitials, resulting in transient enhanced dopant and self-diffusion [7]. In order to isolate the impact of the Fermi level shift on dopant and self-diffusion, the effects of ion implantation must be suppressed. This was achieved by the deposition of a $250 \mathrm{~nm}$ thick amorphous Si cap layer via low temperature molecular beam epitaxy (LT-MBE) on top of the Si isotope heterostructure. The dopant ions were implanted into the amorphous cap layer such that the implantation damage was generated in the cap layer and not within the crystalline isotope heterostructure. Figure 1 shows the results of ${ }^{28} \mathrm{Si}$ implantation into the amorphous cap layer and subsequent annealing. The Si self-diffusion coefficients obtained from fitting the experimental profiles are in excellent agreement with our former results [4]. This shows that ion implantation into the amorphous cap layer and even the re-crystallization of the amorphous layer during annealing does not cause any significant transient enhanced diffusion of $\mathrm{Si}$.

To create the heavily doped arsenic diffusion source sequential implants of arsenic were performed at $130 \mathrm{keV}$ with a dose of $0.7 \times 10^{16} \mathrm{~cm}^{-2}$ and $160 \mathrm{keV}$ at $1.0 \times 10^{16} \mathrm{~cm}^{-2}$ into the cap layer. After implantation, the samples were diced into $4 \times 4 \mathrm{~mm}^{2}$ pieces. Then each individual sample was sealed in a silica ampoule under $0.5 \mathrm{~atm}$ Ar and annealed in a resistance heated furnace at an appropriate time and temperature. The ampoules were quenched in water to 

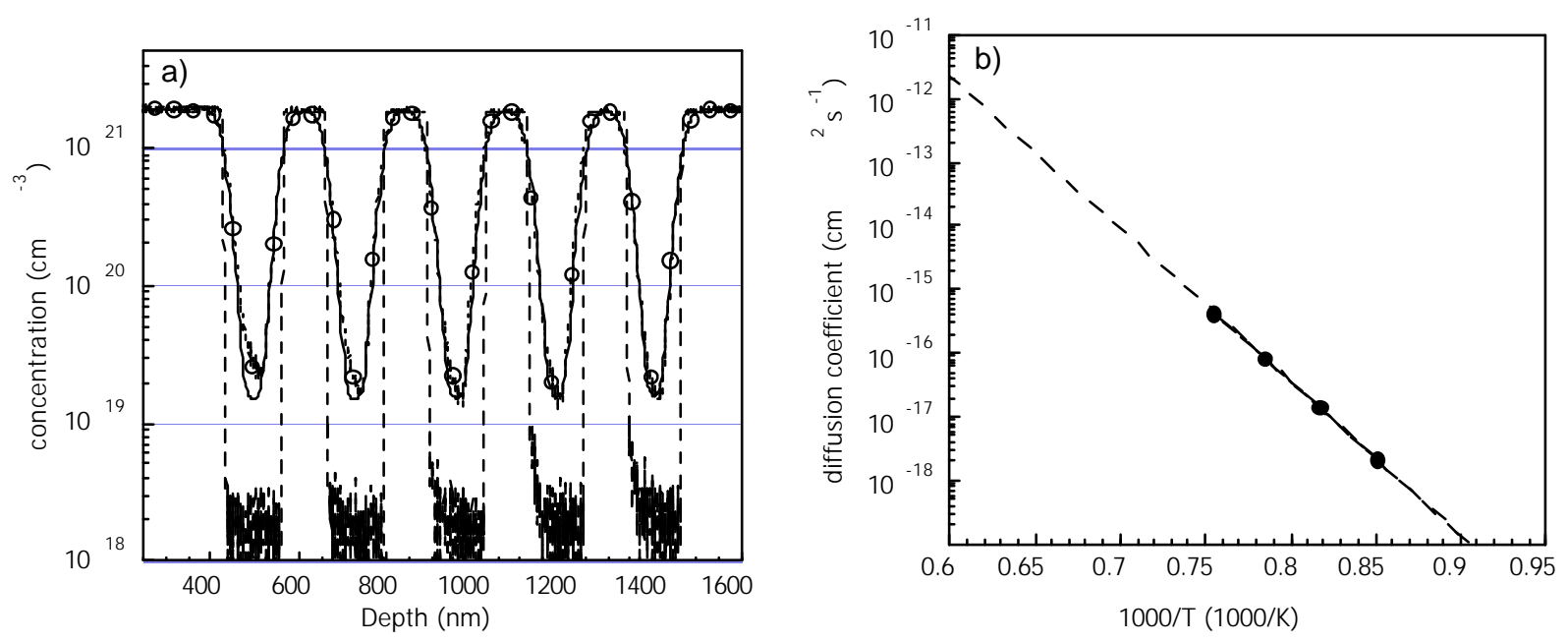

Figure 1 Results of implantation of ${ }^{28} \mathrm{Si}$ into the amorphous Si cap and subsequent anneals. a) SIMS analysis of $1000{ }^{\circ} \mathrm{C}$ annealing for $5 \mathrm{hrs}$ and $17 \mathrm{~min}$. Dashed line represents the preannealed isotope structure, open circles denote the data line and the solid line represents fit to the data. b) Diffusion coefficients determined from fitting the experimental Si profiles which were measured after annealing at various temperatures. The data is in excellent agreement with former results of Si self-diffusion under intrinsic conditions [4] (dashed line).

terminate the diffusion. Depth profiles for ${ }^{30} \mathrm{Si}$ and As were measured via Secondary Ion Mass Spectrometry (SIMS) on an Atomika 4500. A $1.5 \mathrm{keV}$ Cs beam was used for SIMS analysis.

\section{RESULTS}

Arsenic implanted samples were annealed at temperatures between 900 and $1100{ }^{\circ} \mathrm{C}$. Figure 2 illustrates the concentration versus depth profiles of As and $\mathrm{Si}$ after annealing at $950{ }^{\circ} \mathrm{C}$ for $122 \mathrm{hrs}$. Arsenic diffusion is known to take place via interactions with vacancies and interstitials and was modeled as a combination of the vacancy and interstitialcy mechanisms. The experimental As and $\mathrm{Si}$ profiles are mainly described by the reactions:

$$
\begin{aligned}
& (A s V)^{o} \leftrightarrow A s_{s}^{+}+V^{-} \\
& (A s I)^{o} \leftrightarrow A s_{s}^{+}+I^{o}+e^{-}
\end{aligned}
$$

In order to demonstrate the sensitivity of our experimental profiles on the charge state of the native defects, the As and Si profiles were also calculated assuming neutral and doubly negatively charged defects. It is evident from Figure 2 that the enhanced Si self-diffusion within the two near-surface ${ }^{28} \mathrm{Si}$ layers is caused by a singly negatively charged native defect. Note, the As diffusion profile alone is not sensitive to the charge states of the native defects involved. Different charge states for the native defects assumed in the simulation all result in similar As diffusion profiles. This demonstrates that the simultaneous diffusion of dopant-and self-atoms provide a better insight into the mechanisms of dopant diffusion than the analysis of dopant profiles alone. The independence of As diffusion on the charge states of the native defects involved in the diffusion process indicates that the As diffusion is mainly controlled by dopant- 

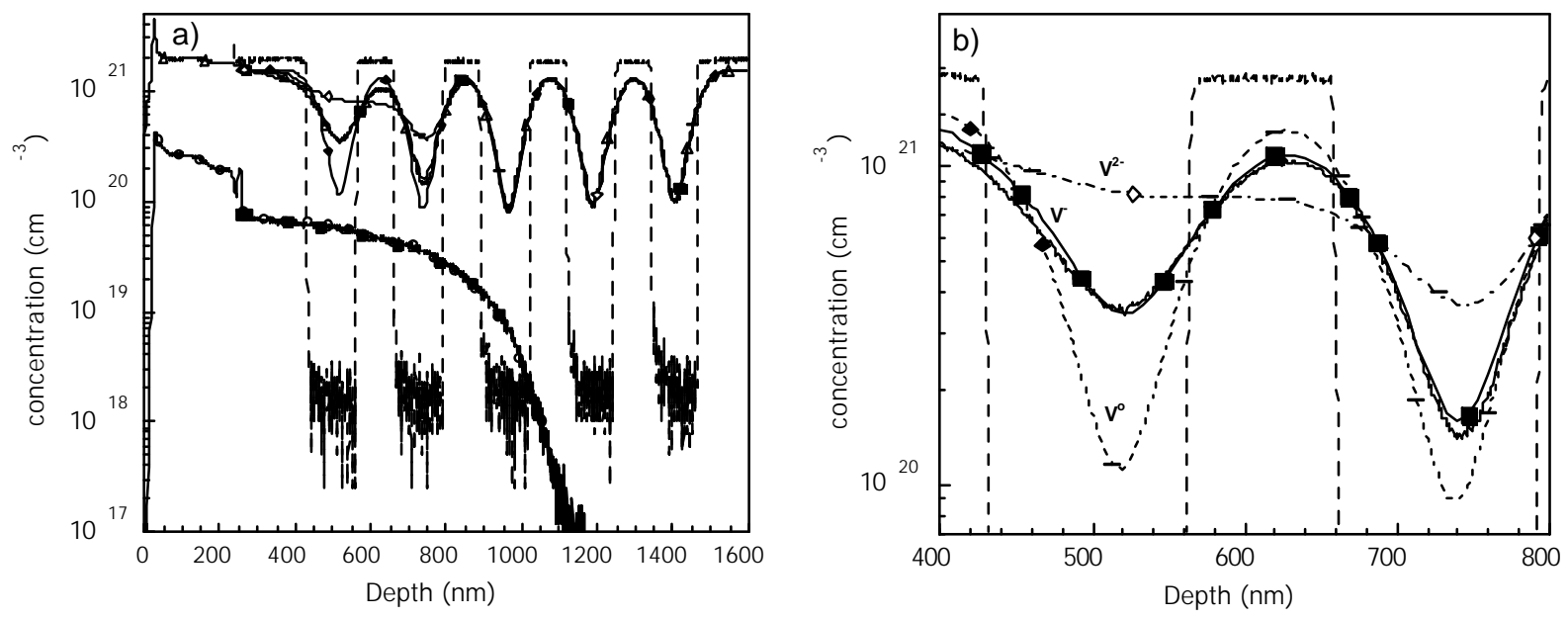

Figure 2 a) Concentration versus depth profile for an As implanted Si isotope heterostructure after annealing at $950 \mathrm{C}$ for 122 hours. Plot shows pre anneal ${ }^{30} \mathrm{Si}$ profile (dashed line) along with the annealed profile for reference. b) Enlarged view of the region of enhanced Si diffusion. Simulated diffusion profiles are overlaid and all vacancy charge state profiles are included to show variation of the simulation with the charge state. $V^{-}$is shown to lead to the best fit to the data (solid line).

defect pairs. Accordingly, As diffusion is not associated with a supersaturation or undersaturation of native point defects which would affect Si self-diffusion. Instead, As diffuses under thermal equilibrium conditions, i.e., the concentration of vacancies and self-interstitials is close to the thermal equilibrium value. Therefore, the enhanced Si diffusion, which is evident from Figure 2 in the region where the As concentration is high, is due to the increased concentration of negatively charged native defects caused by the Fermi level shift. The introduction of the positively charged As ions shifts the Fermi level towards the conduction band, resulting in a lowering of the formation energy for negatively charged defects and an increasing defect concentration.

Finally, it is noted that the native defect favored under n-type doping is assumed to be the singly negatively charged vacancy. On the other hand, the neutral defect is considered to be the self-interstitial. This assignment is consistent with theoretical results describing the charge states of native point defects favored under high n-type doping [8]. However, the experimental As and Si profiles are also accurately described assuming $I^{-}$and $V^{o}$ in reactions 1 and 2, respectively.

\section{DISCUSSION}

The enhancement of diffusion of both the $\mathrm{Si}$ and As under extrinsic conditions is shown in Figure 3(a) in comparison to data for intrinsic conditions (Si:[4]; As:[9]). Similar enhancements of Si diffusion under high n-type doping are reported in the literature [10,11]. The As diffusion coefficients for extrinsic conditions can be reduced to intrinsic conditions via [6]:

$$
D_{A s}\left(n_{i}\right)=D_{A s}(e x t) \cdot \frac{n_{i}}{C_{A s_{s}}^{e q}}
$$


where $n_{i}$ represents the int rinsic carrier concentration [12] and $C_{A s_{s}}^{e q}$ is the concentration of As at the amorphous crystalline interface. The values for $D_{A s}\left(n_{i}\right)$ obtained by Equation (3) are in good agreement with data for $\mathrm{D}_{\mathrm{As}}\left(\mathrm{n}_{\mathrm{i}}\right)$ reported in the literature [9]. This agreement is considered to be a consistency check for the diffusion models which we used to describe the As diffusion. The Si diffusion coefficients under extrinsic condition are mainly determined by the contribution of the negatively charged vacancy.

The individual contributions of the negatively charged vacancy and the neutral interstitial to Si self-diffusion under intrinsic conditions as well as their sum are shown in Figure 3(b). The closed circles in Figure 3(b) represent data from previous diffusion experiments where the total $\mathrm{Si}$ self-diffusion coefficient was determined [4]. The data given by the open symbols represent the contribution of the singly negatively charged vacancy and the neutral self-interstitial, as well as the contribution of the singly positively charged self-interstitial determined from B diffusion experiments [13].

The temperature dependence of the individual contributions to Si self-diffusion and the sum of all contributions follow an Arrhenius expression. The corresponding pre-exponential factors, $\mathrm{D}_{\mathrm{o}}$, and activation enthalpies, $\mathrm{Q}$, are listed in Table 1 . Also included in Table 1 are the values for the total Si self-diffusion determined recently [4].
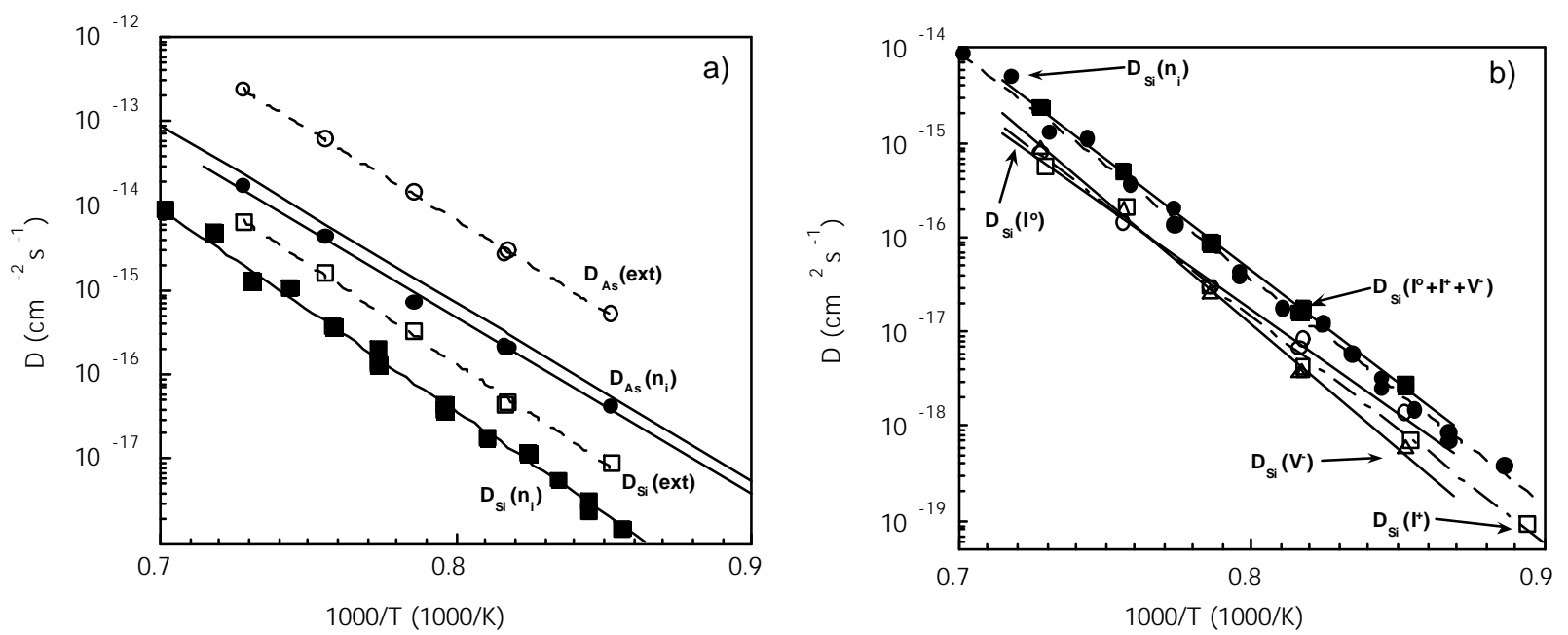

Figure 3 a) Diffusion coefficient versus 1000/T for As (circles) and Si (squares), under intrinsic conditions (solid lines, solid symbols) and extrinsic conditions (dashed lines, open symbols). b) Plot of diffusion coefficient versus 1000/T for Si self-diffusion under intrinsic conditions. The open symbols represent the individual contributions of native defects. The solid squares are the sum of the contributions of the native defects at each temperature. The solid circles are the total self-diffusion values from [4] for comparison.

Table I Prefactor, $\mathrm{D}_{\mathrm{o}}$, and activation energy, Q, for individual native defect contributions to Si self-diffusion and their sum, along with the total Si self-diffusion determined from Ref. [4].

\begin{tabular}{|c|c|c|c|c|c|}
\hline & $I^{o}$ & $I^{+}$ & $V^{-}$ & $D_{\text {sum }}$ & $D_{\mathrm{Si}}$ Ref. 4) \\
\hline $\ln \left(D_{o} / \mathrm{cm}^{2} \mathrm{~s}^{-1}\right)$ & $1.49 \pm 1.37$ & $5.18 \pm 1.24$ & $9.18 \pm 1.19$ & $6.01 \pm 0.76$ & $6.27 \pm 0.39$ \\
\hline $\mathrm{Q}(\mathrm{eV})$ & $4.32 \pm 0.15$ & $4.74 \pm 0.13$ & $5.18 \pm 0.13$ & $4.70 \pm 0.08$ & $4.75 \pm 0.04$ \\
\hline
\end{tabular}




\section{CONCLUSIONS}

The simultaneous diffusion of As and $\mathrm{Si}$ in the isotope multilayer structure is accurately described on the basis of the interstitialcy and vacancy mechanisms. The simulations yield data on the contributions of $V$ and $I^{o}$ to Si self-diffusion. There is no evidence of a $V^{2-}$ component to As diffusion.

The total Si self-diffusion was determined from the sum of the $V^{-}$and $I^{o}$ contributions and the $I^{+}$contribution obtained from B diffusion experiments. This total Si self-diffusion coefficient is in good agreement with data for Si self-diffusion reported recently.

The enhancement of Si self-diffusion during the in-diffusion of As is considered to be associated with the increase in the equilibrium concentration of singly negatively charged vacancies. Negatively charged vacancies are energetically favored when the Fermi level moves towards the conduction band with increasing As doping.

The present study yields an activation enthalpy of Si diffusion via negatively charged vacancies of $5.18 \mathrm{eV}$. The temperature dependence of this contribution suggests that vacancies dominate Si self-diffusion at high temperatures. However, it should be noted that the results deduced for $V^{-}$and $I^{o}$ may also correspond to $I^{-}$and $V^{o}$ because the simulations of the As and $\mathrm{Si}$ diffusion profiles can not distinguish between these native defects.

Additional experiments on the simultaneous diffusion of $\mathrm{P}$ and $\mathrm{Si}$ are in progress that will help to identify the negatively charged native defect under n-type doping conditions.

\section{ACKNOWLEDGEMENTS}

This work was supported in part by industry and the State of California under the UCSMART program No. SM97-01, by US NSF Grant No. DMR-0109844, and by the Director, Office of Science, Office of Basic Energy Sciences, Division of Materials Sciences and Engineering, of the U.S. Department of Energy under Contract No. DE-AC03-76SF00098.

\section{REFERENCES}

1. P.A. Packan, MRS Bulletin 25 (6) 18 (2000).

2. P.M. Fahey, P.B. Griffin, and J.D. Plummer, Reviews of Modern Physics, 61289 (1989).

3. W. Shockley and J.L. Moll, Phys. Rev. 1191480 (1960).

4. H. Bracht, E.E. Haller, and R. Clarke-Phelps, Phys. Rev. Lett. 81393 (1998).

5. A. Ural, P.B. Griffin, and J.D. Plummer, Phys. Rev. Lett. 833454 (1999).

6. M. Uematsu, J. Appl. Phys. 822228 (1997).

7. D.J. Eaglesham, P.A. Stolk, H.-J. Gossmann, J.M. Poate, Appl. Phys. Lett. 65(18) 2305 (1994).

8. P.J. Kelly, R. Car, Phys. Rev. B 456543 (1992).

9. B.J. Masters and J.M. Fairfield, J. Appl. Phys. 402390 (1969).

10. Y. Nakabayashi, H.I. Osman, T. Segawa, K. Saito, S. Matsumoto, J. Murota, K. Wada, T. Abe, Jpn. J. Appl. Phys. 40 L181 (2001).

11. A. Ural, P.B. Griffin, and J.D. Plummer, Appl. Phys. Lett. 794328 (2001).

12. F.J. Morin and J.P. Maita, Phys. Rev. 9628 (1954).

13. I.D. Sharp, et al., (these proceedings). 